\title{
Exponential stability of nonlinear systems with impulsive effects and disturbance input
}

\author{
Xingkai $\mathrm{Hu}^{1,2^{*}}$ and Linru $\mathrm{Nie}^{1,2}$
}

\author{
"Correspondence: \\ huxingkai84@163.com \\ ${ }^{1}$ Faculty of Civil Engineering and \\ Mechanics, Kunming University of \\ Science and Technology, Kunming, \\ P.R. China \\ ${ }^{2}$ Faculty of Science, Kunming \\ University of Science and \\ Technology, Kunming, P.R. China
}

\begin{abstract}
In this paper, exponential stability of nonlinear systems with impulse time window, disturbance input and bounded gain error is investigated. By means of the above result and the construction of a linear stabilizing feedback controller, another criterion of exponential stability is established. A numerical example is given to demonstrate the effectiveness of the theoretical results.
\end{abstract}

MSC: $37 N 35 ; 49 N 25$

Keywords: Exponential stability; Impulse time window; Disturbance input; Bounded gain error; Linear matrix inequalities

\section{Introduction}

Customarily, $R_{+}$denotes the set of positive real numbers. $R^{n}$ is an $n$-dimensional real Euclidean space with the norm $\|\cdot\| \cdot R^{m \times n}$ refers to the set of all $m \times n$-dimensional real matrices. $\lambda_{M}(A), \lambda_{m}(A), A^{T}$, and $A^{-1}$ are the maximum, the minimum eigenvalue, the transpose, and the inverse of matrix $A$, respectively. $I$ represents the identity matrix with proper dimension. The positive definite matrix $A$ is represented by $A>0$. Define $f\left(x\left(b^{-}\right)\right)=\lim _{t \rightarrow b^{-}} f(x(t))$.

Over the past two decades, nonlinear systems have been paid considerable attention because many systems in many practical applications can be modeled by nonlinear systems, for instance, robotics, information science, artificial intelligence, automatic control systems, and so forth $[8,14,15,17,24]$. Due to impulsive effects, the stability of systems will become oscillations and instability. Therefore, it is significant to discuss stability of nonlinear systems with impulsive effects $[9,10,19,21,22]$. In recent years, many sufficient criteria on the asymptotic stability for impulsive control of nonlinear systems have been published under some conditions $[1,16]$. We consider not only the asymptotic satiability of the nonlinear impulsive control systems but other aspects in the design of nonlinear impulsive control systems. In particular, it is often desirable that nonlinear impulsive control systems converge fast enough in order to reach fast response. Obviously, exponential stability is a fast convergence rate to the equilibrium point $[7,11,13]$.

Many scholars just assume that impulses occur at fixed-time points [12, 18, 20]. However, in many practical applications, impulses occur stochastically. Therefore, it is necessary to study a more practical impulsive scheme which concerns the above case. In what follows, we will discuss the following nonlinear impulsive control systems with impulse

(c) The Author(s) 2018. This article is distributed under the terms of the Creative Commons Attribution 4.0 International License (http://creativecommons.org/licenses/by/4.0/), which permits unrestricted use, distribution, and reproduction in any medium, provided you give appropriate credit to the original author(s) and the source, provide a link to the Creative Commons license, and indicate if changes were made. 
time window, disturbance input and bounded gain error:

$$
\begin{cases}\dot{x}(t)=A x(t)+B w(t)+C u(t)+f(x(t)), & k T \leq t<k T+\tau_{k}, \\ x(t)=x\left(t^{-}\right)+Q x\left(t^{-}\right)+\phi\left(x\left(t^{-}\right)\right), & t=k T+\tau_{k}, \\ \dot{x}(t)=A x(t)+B w(t)+C u(t)+f(x(t)), & k T+\tau_{k}<t<(k+1) T,\end{cases}
$$

where $x(t) \in R^{n}$ is the state variable, $w(t) \in R^{r}$ denotes the disturbance input, $u(t) \in R^{p}$ is the control input, $\phi(x(t))$ is the gain error, $f: R^{n} \rightarrow R^{n}$ and $\phi: R^{n} \rightarrow R^{n}$ are said to be continuous nonlinear functions satisfying $f(0)=0$ and $\phi(0)=0$, respectively, $T>0$ represents the control period, $\tau_{k} \in(k T,(k+1) T)$ is unknown. $A \in R^{n \times n}, B \in R^{n \times r}, C \in R^{n \times p}$, and $Q \in R^{n \times n}$ are constant matrices. In general, let

$$
\begin{aligned}
& \|f(x(t))\| \leq l\|x(t)\|, \\
& \|w(t)\| \leq l_{1}\|x(t)\|, \\
& \|\phi(x(t))\| \leq l_{2}\|x(t)\|,
\end{aligned}
$$

where $l, l_{1}$, and $l_{2}$ are nonnegative constants. In system (1.1), the impulse is stochastic in an impulse time window, which is wider than an impulse occurring at fixed-time points. For more information on an impulse time window, the reader is referred to [3-5, 23].

In order to obtain exponential stability, a linear feedback controller $u(t)=G x(t)$ is considered, where $G \in R^{r \times n}$ is a constant matrix. We rewrite system (1.1) as follows:

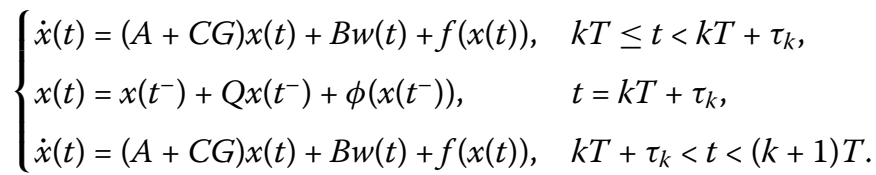

The main purpose of this paper is to investigate the exponential stability of system (1.1). By employing the obtained result, system (1.2) is exponentially stable via constructing a linear feedback gain matrix $G$. A numerical example is given to demonstrate the effectiveness of the theoretical results.

\section{Main results}

We need the following definitions and lemmas which play a major role in the proof of the theorems.

Definition 2.1 ([11]) The function $V:\left[t_{0}-\alpha, \infty\right) \times R^{n} \rightarrow R_{+}$belongs to class $v_{0}$ if

(1) $V$ is continuous on each of the sets $\left[\tau_{k-1}, \tau_{k}\right) \times R^{n}$ and $\lim _{(t, y) \rightarrow\left(\tau_{k}^{-}, x\right)} V(t, y)=V\left(\tau_{k}^{-}, x\right)$ exists;

(2) $V(t, x)$ is locally Lipschitzian in $x \in R^{n}$ and $V(t, 0) \equiv 0$.

Definition 2.2 ([11]) For $V \in v_{0}$, the right and upper Dini's derivative of $V$ is defined as

$$
D^{+} V(t, x(t))=\lim _{h \rightarrow 0^{+}} \sup \frac{1}{h}[V(t+h, x(t)+h f(t, x(t)))-V(t, x(t))] .
$$


Lemma 2.1 ([6]) Let $x, y \in R^{n}$ and $\eta>0$, then

$$
2 x^{T} y \leq \eta x^{T} x+\eta^{-1} y^{T} y .
$$

Lemma 2.2 ([2]) The following linear matrix inequality (LMI)

$$
\left[\begin{array}{cc}
Q & S \\
S^{T} & G
\end{array}\right]<0
$$

where $Q^{T}=Q, G^{T}=G$, is equivalent to

$$
G<0, \quad Q-S G^{-1} S^{T}<0 .
$$

Lemma 2.3 ([6]) Let $x \in R^{n}$ and $A \in R^{n \times n}$ be a symmetric matrix, then

$$
\lambda_{m}(A) x^{T} x \leq x^{T} A x \leq \lambda_{M}(A) x^{T} x .
$$

Theorem 2.1 Let the assumptions about $w(t), f(x(t)), \phi(x(t))$ be satisfied and $u(t)=0$. If there exist positive numbers $\varepsilon, \eta$ and $0<P \in R^{n \times n}$ satisfying conditions as follows:
(1) $\left[\begin{array}{cc}A^{T} P+P A+\left(l^{2}+\eta l_{1}^{2}\right) I & P \\ P & \left(-I-\eta^{-1} B B^{T}\right)^{-1}\end{array}\right]<0$,
(2) $\ln \gamma+T(h+\varepsilon) \leq 0$,

where $\beta=\lambda_{M}\left(P^{-1}(I+Q)^{T} P(I+Q)\right), \beta_{1}=\lambda_{M}(P), \beta_{2}=\lambda_{m}(P), h=\lambda_{M}\left(P^{-1}\left(P A+A^{T} P+\right.\right.$ $\left.\left.\eta^{-1} P B B^{T} P+P^{2}+\left(l^{2}+\eta l_{1}^{2}\right) I\right)\right), \gamma=\left(\sqrt{\beta}+\sqrt{\frac{\beta_{2}}{\beta_{3}}} l_{2}\right)^{2}$. Then system (1.1) is exponentially stable at origin.

Proof Define

$$
V(x(t))=x^{T}(t) P x(t)
$$

Let $t \in\left[k T, k T+\tau_{k}\right)$, we have

$$
\begin{aligned}
D^{+}(V(x(t))) & =2 x^{T}(t) P(A x(t)+B w(t)+f(x(t))) \\
& =x^{T}(t)\left(P A+A^{T} P\right) x(t)+2 x^{T}(t) P(B w(t)+f(x(t))) .
\end{aligned}
$$

By Lemma 2.1, it is clear that

$$
2 x^{T}(t) P B w(t) \leq \eta^{-1} x^{T}(t) P B B^{T} P x(t)+\eta w^{T}(t) w(t)
$$

and

$$
2 x^{T}(t) P f(x(t)) \leq x^{T}(t) P^{2} x(t)+f^{T}(x(t)) f(x(t)) .
$$


From the assumptions about $f(x(t)), w(t)$, substituting (2.2) and (2.3) into (2.1) yields

$$
\begin{aligned}
D^{+}(V(x(t))) \leq & x^{T}(t)\left(P A+A^{T} P\right) x(t)+\eta^{-1} x^{T}(t) P B B^{T} P x(t) \\
& +\eta w^{T}(t) w(t)+x^{T}(t) P^{2} x(t)+f^{T}(x(t)) f(x(t)) \\
\leq & x^{T}(t)\left(P A+A^{T} P+\eta^{-1} P B B^{T} P+P^{2}+\left(l^{2}+\eta l_{1}^{2}\right) I\right) x(t) .
\end{aligned}
$$

By Lemma 2.2, condition (1) and inequality (2.4), we have

$$
D^{+}(V(x(t))) \leq h V(x(t))
$$

which yields that

$$
V(x(t)) \leq V(x(k T)) e^{h(t-k T)}
$$

In the same way, let $t \in\left(k T+\tau_{k},(k+1) T\right)$, we also have

$$
D^{+}(V(x(t))) \leq h V(x(t))
$$

which leads to

$$
V(x(t)) \leq V\left(x\left(k T+\tau_{k}\right)\right) e^{h\left(t-k T-\tau_{k}\right)}
$$

Let $t=k T+\tau_{k}$, we obtain

$$
\begin{aligned}
V(x(t))= & \left((I+Q) x\left(t^{-}\right)+\phi\left(x\left(t^{-}\right)\right)\right)^{T} P\left((I+Q) x\left(t^{-}\right)+\phi\left(x\left(t^{-}\right)\right)\right) \\
= & x^{T}\left(t^{-}\right)(I+Q)^{T} P(I+Q) x\left(t^{-}\right)+\phi^{T}\left(x\left(t^{-}\right)\right) P \phi\left(x\left(t^{-}\right)\right) \\
& +2 x^{T}\left(t^{-}\right)(I+Q)^{T} P \phi\left(x\left(t^{-}\right)\right) \\
\leq & x^{T}\left(t^{-}\right)(I+Q)^{T} P(I+Q) x\left(t^{-}\right)+\phi^{T}\left(x\left(t^{-}\right)\right) P \phi\left(x\left(t^{-}\right)\right) \\
& +2 \sqrt{x^{T}\left(t^{-}\right)(I+Q)^{T} P(I+Q) x\left(t^{-}\right) \phi^{T}\left(x\left(t^{-}\right)\right) P \phi\left(x\left(t^{-}\right)\right)} \\
= & \left(\sqrt{x^{T}\left(t^{-}\right)(I+Q)^{T} P(I+Q) x\left(t^{-}\right)}+\sqrt{\phi^{T}\left(x\left(t^{-}\right)\right) P \phi\left(x\left(t^{-}\right)\right)}\right)^{2} \\
\leq & \left(\sqrt{\beta}+\sqrt{\frac{\beta_{2}}{\beta_{3}}} l_{2}\right)^{2} V\left(x\left(t^{-}\right)\right) \\
= & \gamma V\left(x\left(t^{-}\right)\right) .
\end{aligned}
$$

(2.6) and (2.7) can lead to

$$
V(x(t)) \leq \gamma V\left(x\left(\left(k T+\tau_{k}\right)^{-}\right)\right) e^{h\left(t-k T-\tau_{k}\right)},
$$

where $t \in\left[k T+\tau_{k},(k+1) T\right)$.

When $k=0$, let $t \in\left[0, \tau_{0}\right)$, from (2.5), we obtain

$$
V(x(t)) \leq V(x(0)) e^{h t}
$$


Thus

$$
V\left(x\left(\tau_{0}^{-}\right)\right) \leq V(x(0)) e^{h \tau_{0}} .
$$

Let $t \in\left[\tau_{0}, T\right)$, from (2.8) and (2.9), we have

$$
V(x(t)) \leq \gamma V\left(x\left(\tau_{0}^{-}\right)\right) e^{h\left(t-\tau_{0}\right)} \leq \gamma V(x(0)) e^{h t} .
$$

When $k=1$, let $t \in\left[T, T+\tau_{1}\right)$, from (2.5) and (2.10), we have

$$
\begin{aligned}
V(x(t)) & \leq V(x(T)) e^{h(t-T)} \\
& \leq \gamma V\left(x\left(\tau_{0}^{-}\right)\right) e^{h\left(T-\tau_{0}\right)} e^{h(t-T)} \\
& =\gamma V\left(x\left(\tau_{0}^{-}\right)\right) e^{h\left(t-\tau_{0}\right)} \\
& \leq \gamma V(x(0)) e^{h t} .
\end{aligned}
$$

Let $t \in\left[T+\tau_{1}, 2 T\right)$, from (2.8) and (2.11), we get

$$
\begin{aligned}
V(x(t)) & \leq \gamma V\left(x\left(\left(T+\tau_{1}\right)^{-}\right)\right) e^{h\left(t-T-\tau_{1}\right)} \\
& \leq \gamma^{2} V\left(x\left(\tau_{0}^{-}\right)\right) e^{h\left(T+\tau_{1}-\tau_{0}\right)} e^{h\left(t-T-\tau_{1}\right)} \\
& \leq \gamma^{2} V(x(0)) e^{h t} .
\end{aligned}
$$

When $k=2$, let $t \in\left[2 T, 2 T+\tau_{2}\right)$, from (2.5) and (2.12), we get

$$
\begin{aligned}
V(x(t)) & \leq V(x(2 T)) e^{h(t-2 T)} \\
& \leq \gamma^{2} V\left(x\left(\tau_{0}^{-}\right)\right) e^{h\left(2 T-\tau_{0}\right)} e^{h(t-2 T)} \\
& \leq \gamma^{2} V(x(0)) e^{h t} .
\end{aligned}
$$

Let $t \in\left[\tau_{0}, T+\tau_{1}\right)$, from (2.10) and (2.11), we get

$$
V(x(t)) \leq \gamma V(x(0)) e^{h t} .
$$

Let $t \in\left[T+\tau_{1}, 2 T+\tau_{2}\right)$, from (2.12) and (2.13), we get

$$
V(x(t)) \leq \gamma^{2} V(x(0)) e^{h t} .
$$

By induction, for $t \in\left[k T+\tau_{k},(k+1) T+\tau_{k+1}\right)$, we get

$$
V(x(t)) \leq \gamma^{k+1} V(x(0)) e^{h t} .
$$

Let $k T+\tau_{k}=\tau_{k}^{\prime}$. Since $\ln \gamma+T(h+\varepsilon) \leq 0$, we get

$$
\begin{aligned}
V(x(t)) & \leq \gamma^{k+1} V(x(0)) e^{h t} \\
& =\gamma^{k+1} V(x(0)) e^{(h+\varepsilon) t} e^{-\varepsilon t}
\end{aligned}
$$




$$
\begin{aligned}
& \leq \gamma^{k+1} V(x(0)) e^{(h+\varepsilon) \tau_{k}^{\prime}} e^{-\varepsilon t} \\
& \leq \gamma^{k+1} V(x(0)) e^{(h+\varepsilon) k T} e^{-\varepsilon t} \\
& =\gamma V(x(0)) e^{k(\ln \gamma+(h+\varepsilon) T)} e^{-\varepsilon t} \\
& \leq \gamma V(x(0)) e^{-\varepsilon t} .
\end{aligned}
$$

By Lemma 2.3 and (2.14), we obtain

$$
\lambda_{m}(P)\left\|x\left(t, \tau_{0}, x(0)\right)\right\|^{2} \leq V(x(t)) \leq \gamma V(x(0)) e^{-\varepsilon t} \leq\|x(0)\|^{2} \lambda_{M}(P) e^{-\varepsilon t} .
$$

That is,

$$
\left\|x\left(t, \tau_{0}, x(0)\right)\right\| \leq \sqrt{\frac{\lambda_{M}(P)}{\lambda_{m}(P)}}\|x(0)\| e^{\frac{-\varepsilon t}{2}} .
$$

This completes the proof.

Theorem 2.2 Let the assumptions about $w(t), f(x(t)), \phi(x(t))$ be satisfied. If there exist positive numbers $\varepsilon, \eta$, matrices $H, W$ with $0<H \in R^{n \times n}$ satisfying conditions as follows:

$$
\begin{aligned}
& \text { (1) }\left[\begin{array}{cc}
I+(A H+C W)^{T}+(A H+C W)+\eta^{-1} B B^{T} & \sqrt{l^{2}+\eta l_{1}^{2}} H \\
\sqrt{l^{2}+\eta l_{1}^{2}} H & -I
\end{array}\right]<0, \\
& \text { (2) } \sqrt{\beta}+\sqrt{\frac{\beta_{2}}{\beta_{3}}} l_{2}<1,
\end{aligned}
$$

where $\beta=\lambda_{M}\left(H(I+Q)^{T} H^{-1}(I+Q)\right), \beta_{1}=\lambda_{M}\left(H^{-1}\right), \beta_{2}=\lambda_{m}\left(H^{-1}\right), h=\lambda_{M}\left(H\left(H^{-1}(A+\right.\right.$ $\left.\left.C G)+(A+C G)^{T} H^{-1}+\eta^{-1} H^{-1} B B^{T} H^{-1}+\left(H^{-1}\right)^{2}+\left(l^{2}+\eta l_{1}^{2}\right) I\right)\right), \gamma=\left(\sqrt{\beta}+\sqrt{\frac{\beta_{2}}{\beta_{3}}} l_{2}\right)^{2}$. Then system (1.2) is exponentially stable at origin and we have the following linear feedback controller:

$$
u(t)=G x(t), \quad G=W H^{-1} .
$$

Proof By Lemma 2.2, condition (1) of Theorem 2.2 is equivalent to

$$
I+(A H+C W)^{T}+(A H+C W)+\eta^{-1} B B^{T}+\left(l^{2}+\eta l_{1}^{2}\right) H^{2}<0 .
$$

Let

$$
P=H^{-1}, \quad G=W H^{-1} .
$$

Multiplying both sides of (2.15) by $P$, we have

$$
P^{2}+P(A H+C W)^{T} P+P(A H+C W) P+\eta^{-1} P B B^{T} P+\left(l^{2}+\eta l_{1}^{2}\right) I<0 .
$$

That is,

$$
P^{2}+(A+C G)^{T} P+P(A+C G)+\eta^{-1} P B B^{T} P+\left(l^{2}+\eta l_{1}^{2}\right) I<0 .
$$


By Lemma 2.2 and (2.16), we have

$$
\left[\begin{array}{cc}
(A+C G)^{T} P+P(A+C G)+\left(l^{2}+\eta l_{1}^{2}\right) I & P \\
P & \left(-I-\eta^{-1} B B^{T}\right)^{-1}
\end{array}\right]<0 .
$$

Thus, condition (1) of Theorem 2.1 holds. Since

$$
\sqrt{\beta}+\sqrt{\frac{\beta_{2}}{\beta_{3}}} l_{2}<1
$$

which implies

$$
\ln \gamma+T(h+\varepsilon) \leq 0
$$

namely, condition (2) of Theorem 2.1 is satisfied, too. Then system (2.2) is exponentially stable at origin.

This completes the proof.

\section{A numerical example}

In this section, we demonstrate and verify the effectiveness of our theoretical results employing a nonlinear impulsive system as follows:

$$
A=\left[\begin{array}{ll}
2 & 1 \\
2 & 3
\end{array}\right], \quad B=\left[\begin{array}{cc}
1.5 & 1.3 \\
1.2 & 0
\end{array}\right], \quad C=\left[\begin{array}{cc}
1.8 & 0.8 \\
1 & 1.7
\end{array}\right], \quad f(x(t))=\left[\begin{array}{l}
\sin x_{1} \\
\sin x_{2}
\end{array}\right]
$$

and

$$
Q=-\left[\begin{array}{cc}
0.58 & 0 \\
0 & 0.58
\end{array}\right], \quad \phi(x(t))=0.3\left[\begin{array}{l}
\sin x_{1} \\
\sin x_{2}
\end{array}\right], \quad \omega(t)=\left[\begin{array}{l}
x_{1} \sin 20 \pi t \\
x_{2} \sin 20 \pi t
\end{array}\right] .
$$

Then we can choose

$$
\eta=l=l_{1}=1, \quad l_{2}=0.3 .
$$

By condition (1) of Theorem 2.2, we obtain

$$
H=\left[\begin{array}{cc}
0.2565 & 0 \\
0 & 0.2565
\end{array}\right], \quad W=\left[\begin{array}{cc}
-25.7414 & -49.9086 \\
53.3876 & 27.8104
\end{array}\right] .
$$

Simple calculations show that $\gamma=0.72<1$. Thus, the nonlinear impulsive system is exponentially stable because the conditions of Theorem 2.2 are satisfied.

\section{Conclusions}

In this paper, we discuss exponential stability of nonlinear systems with impulse time window, disturbance input, and bounded gain error. In [3], the authors did not consider the disturbance input and bounded gain error of nonlinear impulsive control systems. In [25], the authors did not consider the disturbance input of nonlinear impulsive control systems. 
Obviously, system (1.1) is more general and more applicable than [3, 25]. Using Theorem 2.1 and the construction of a linear stabilizing feedback controller, a new criterion of exponential stability is obtained. Finally, a numerical example demonstrates the effectiveness of the theoretical results.

\section{Acknowledgements}

The authors would like to express their sincere thanks to referees and the editor for their enthusiastic guidance and help.

\section{Funding}

This research is supported by the National Natural Science Foundation of China (Grant Nos. 11561037, 11801240).

\section{Competing interests}

The authors declare that they have no competing interests.

\section{Authors' contributions}

All authors contributed equally to the manuscript. All authors read and approved the final manuscript.

\section{Publisher's Note}

Springer Nature remains neutral with regard to jurisdictional claims in published maps and institutional affiliations.

Received: 14 July 2018 Accepted: 10 September 2018 Published online: 04 October 2018

\section{References}

1. Ai, Z., Chen, C.: Asymptotic stability analysis and design of nonlinear impulsive control systems. Nonlinear Anal. Hybrid Syst. 24, 244-252 (2017)

2. Boyd, S., Ghaoui, E.I.L., Feron, E., Balakrishnan, V.: Linear Matrix Inequalities in System and Control Theory. SIAM, Philadelphia (1994)

3. Feng, Y., Li, C., Huang, T.: Periodically multiple state-jumps impulsive control systems with impulse time windows. Neurocomputing 193, 7-13 (2016)

4. Feng, Y., Li, C., Huang, T.: Sandwich control systems with impulse time windows. Int. J. Mach. Learn. Cybern. 8 2009-2015 (2017)

5. Feng, Y., Peng, Y., Zou, L., Tu, Z., Liu, J.: A note on impulsive control of nonlinear systems with impulse time window. J. Nonlinear Sci. Appl. 10, 3087-3098 (2017)

6. Horn, R.A., Johnson, C.R.: Matrix Analysis. Cambridge University Press, Cambridge (1985)

7. Huang, T., Li, C., Duan, S., Starzyk, J.A.: Robust exponential stability of uncertain delayed neural networks with stochastic perturbation and impulse effects. IEEE Trans. Neural Netw. Learn. Syst. 23, 866-875 (2012)

8. Li, X., Bohner, M., Wang, C.: Impulsive differential equations: periodic solutions and applications. Automatica 52 $173-178(2015)$

9. Li, X., Cao, J.: An impulsive delay inequality involving unbounded time-varying delay and applications. IEEE Trans. Autom. Control 62, 3618-3625 (2017)

10. Li, X., Song, S.: Stabilization of delay systems: delay-dependent impulsive control. IEEE Trans. Autom. Control 62 , 406-411 (2017)

11. Li, X., Wu, J.: Stability of nonlinear differential systems with state-dependent delayed impulses. Automatica 64,63-69 (2016)

12. Li, Z., Wen, C., Soh, Y.: Analysis and design of impulsive control systems. IEEE Trans. Autom. Control 46, 894-897 (2001)

13. Song, Q., Cao, J.: Global exponential stability of bidirectional associative memory neural networks with distributed delays. J. Comput. Appl. Math. 202, 266-279 (2007)

14. Song, Q., Cao, J.: Passivity of uncertain neural networks with both leakage delay and time-varying delay. Nonlinear Dyn. 67, 169-1707 (2012)

15. Song, Q., Zhao, Z.: Stability criterion of complex-valued neural networks with both leakage delay and time-varying delays on time scales. Neurocomputing 171, 179-184 (2016)

16. Sun, J., Wu, Q.: Impulsive control for the stabilization and synchronization of Lorenz systems. Appl. Math. Mech. 25, 322-328 (2004)

17. Wang, H., Liao, X., Huang, T., Li, C.: Improved weighted average prediction for multi-agent networks. Circuits Syst. Signal Process. 33, 1721-1736 (2014)

18. Yang, T.: Impulsive control. IEEE Trans. Autom. Control 44, 1081-1083 (1999)

19. Yang, X., Feng, Z., Feng, J., Cao, J.: Synchronization of discrete-time neural networks with delays and Markov jump topologies based on tracker information. Neural Netw. 85, 157-164 (2017)

20. Yang, X, Lam, J.,Ho, D.W.C. Feng, Z: Fixed-time synchronization of complex networks with impulsive effects via nonchattering control. IEEE Trans. Autom. Control 62, 5511-5521 (2017)

21. Yang, Z., Xu, D.: Stability analysis and design of impulsive control systems with time delay. IEEE Trans. Autom. Control $52,1448-1454$ (2007)

22. Zhang, Y.: Stability of discrete-time Markovian jump delay systems with delayed impulses and partly unknown transition probabilities. Nonlinear Dyn. 75, 101-111 (2014)

23. Zhou, Y., Li, C., Huang, T., Wang, X.: Impulsive stabilization and synchronization of Hopfield-type neural networks with impulse time window. Neural Comput. Appl. 28, 775-782 (2017)

24. Zou, L., Peng, Y., Feng, Y., Tu, Z.: Stabilization and synchronization of memristive chaotic circuits by impulsive control. Complexity 2017, Article ID 5186714 (2017)

25. Zou, L., Peng, Y., Feng, Y., Tu, Z.: Impulsive control of nonlinear systems with impulse time window and bounded gain error. Nonlinear Anal., Model. Control 23, 40-49 (2018) 